\title{
Far-infrared spectroscopy of $\mathrm{CO}_{2}$ clathrate hydrate with Martian applications
}

\author{
Joseph C. Landry and Anthony W. England ${ }^{1}$ \\ Radiation Laboratory, Electrical Engineering and Computer Science Dept., University of Michigan
}

\begin{abstract}
It has been suggested that $\mathrm{CO}_{2}$ clathrate hydrate forms in the Martian polar regions in winter. A large deposit of hydrate would affect the polar energy budget because of its low thermal conductivity and its sizeable latent heat of dissociation, and it could also be important to the total $\mathrm{CO}_{2}$ budget. In this paper we present the far-infrared spectrum of $\mathrm{CO}_{2}$ hydrate at $150 \mathrm{~K}$. The spectrum exhibits a broad absorption centered near $17 \mathrm{~cm}^{-1}(0.59 \mathrm{~mm})$ that is probably due to a rattling transition. Our radiative transfer model predicts that the absorption gives rise to a distinctive emission spectrum for densely packed particles having average diameters greater than $100 \mu \mathrm{m}$.
\end{abstract}

\section{Introduction}

If liquid water is sufficiently pressurized with $\mathrm{CO}_{2}$ and cooled, it will crystallize into a solid called a clathrate hydrate. The general clathrate hydrate structure consists of an $\mathrm{H}_{2} \mathrm{O}$ host lattice with voids that encage individual guest molecules. In the case of $\mathrm{CO}_{2}$, the $\mathrm{H}_{2} \mathrm{O}$ lattice forms a structure I clathrate hydrate, which has two sizes of cages accommodating molecules up to 0.51 $\mathrm{nm}$ and $0.58 \mathrm{~nm}$ in diameter. $\mathrm{CO}_{2}$ guest molecules stabilize the cages, which otherwise would collapse due to attractive forces. As long as the $\mathrm{CO}_{2}$ partial pressure is above the hydrate dissociation pressure, the hydrate is more stable than a water ice $+\mathrm{CO}_{2}$ gas system.

Other naturally abundant molecules in the solar system, such as $\mathrm{CH}_{4}, \mathrm{~N}_{2}$ and $\mathrm{Ar}$, can combine with water to form clathrate hydrates. Besides Earth, Miller [1985] lists Uranus, Neptune, Titan, Mars and comets as likely sites for hydrate formation. We concentrate on the Martian $\mathrm{CO}_{2}$ problem, but the techniques developed here should be applicable to the study of other hydrate scenarios.

The polar regions of Mars undergo profound seasonal changes [James et al., 1992]. During the northern polar winter, $\mathrm{CO}_{2}$ condenses to form a seasonal cap. In the spring, it retreats to expose a permanent water ice cap.

\footnotetext{
${ }^{1}$ Also at Atmospheric, Oceanic and Space Sciences Dept., University of Michigan
}

Copyright 1994 by the American Geophysical Union.

Paper number 94GL02694

0094-8534/94/94GL-02694\$03.00
Since the temperature for stability of $\mathrm{CO}_{2}$ hydrate is higher than that of pure $\mathrm{CO}_{2}$ ice, some of the water ice may convert to hydrate during the winter, and this hydrate may remain for some time after the seasonal cap has retreated. A similar seasonal cap forms in the southern hemisphere, but the south residual cap is $\mathrm{CO}_{2}$ ice. If a water ice cap is beneath the $\mathrm{CO}_{2}$ cap, it is probably not remotely detectable.

The presence of $\mathrm{CO}_{2}$ hydrate on Mars is important because it may significantly affect the energy budget. The thermal conductivity of other structure I hydrates are significantly lower than that of pure water ice [Handa, 1987]. A hydrate deposit could therefore be a more effective insulator than an ice deposit. Furthermore, the latent heat of dissociation is $5.86 \mathrm{kcal} / \mathrm{mol}$ [Davidson, 1973, p. 166], which is about half the latent heat of sublimation of water ice and about equal to that of $\mathrm{CO}_{2}$ ice. If a large portion of either cap is hydrate, then a significant amount of energy is released when it forms and is consumed when it dissociates. $\mathrm{CO}_{2}$ hydrate in the polar caps would also affect the total budget of $\mathrm{CO}_{2}$ in the atmosphere-polar cap-regolith system.

To detect $\mathrm{CO}_{2}$ hydrate through a radiometric measurement, its dielectric properties must be sufficiently different from those of other environmental constituents to yield an unambiguous signature. The hydrogenbonded host lattice of structure I hydrates is similar to that of ice Ic. Studies of ethylene oxide hydrate [Bertie et al., 1973] and cubic ice [Bertie and Whalley, 1964, 1967] in the range $100-4000 \mathrm{~cm}^{-1}$ show quite similar absorption spectra. Furthermore, Smythe [1975] found virtually no difference between the reflectance spectra of water ice frost and $\mathrm{CO}_{2}$ and $\mathrm{CH}_{4}$ hydrate frosts in the range 1-6 $\mu \mathrm{m}$. Some authors have pointed out spectral differences, such as the broadening and slight shifting of the $\mathrm{O}-\mathrm{H}$ stretching mode absorption in various hydrate spectra when compared to the water ice spectrum [Richardson et al., 1985]. However, the differences are subtle and not easily detected in remotely sensed data.

Guests in clathrate hydrates are not held by bonds to the water lattice, but instead are trapped by the potential barrier of the cage walls. A guest has some freedom to rotate, vibrate, and rattle within its cage, which gives rise to infrared absorption. Due to the near spherical shape of the structure I cages, guest rotation is largely unconstrained and is close to that of the free molecule, though it is affected by collisions with the cage walls. Since $\mathrm{CO}_{2}$ has no pure rotational transitions, the encaged molecule should not exhibit any 
strong rotational absorptions. The fundamental vibrational absorptions of the encaged $\mathrm{CO}_{2}$ molecule have been measured [Fleyfel and Devlin, 1988] and occur in the same region as the vibrational spectrum of $\mathrm{CO}_{2}$ gas [Aldrich Library of FT-IR Spectra, 1989, p. 759] and $\mathrm{CO}_{2}$ ice [Warren, 1986]. We are unaware of any measurements of the $\mathrm{CO}_{2}$ overtone or combinational bands, but we believe they will also occur within regions of gas and ice absorption. Hence, $\mathrm{CO}_{2}$ vibrational absorptions will probably not produce a distinctive radiometric signature. Furthermore, observations of hydrate on Mars at the vibrational absorption frequencies will be obscured by atmospheric $\mathrm{CO}_{2}$ vibrational absorption.

Rattling mode absorptions are more promising since there is no analogous mechanism for absorption in water ice or in $\mathrm{CO}_{2}$ gas or ice. Such absorptions could provide a distinctive signature. Rattling absorptions always occur in the far-infrared, typically below $100 \mathrm{~cm}^{-1}$. Their behavior has been simulated by Tse et al. [1984]. This paper presents far-infrared absorption spectra of $\mathrm{CO}_{2}$ hydrate showing a broad absorption peak at $17 \mathrm{~cm}^{-1}$ that is probably due to a rattling transition.

\section{Experimental}

Other investigators have formed clathrate hydrates by depositing mixtures of water vapor and the guest species on a cooled substrate [e.g., Fleyfel and Devlin, 1988] or by pressurizing liquid water or ice with the guest species [e.g., Kiefte, 1985]. In general, they found that hydrates with a polar guest have lower dissociation pressures and form more quickly. One way to expedite formation with a non-polar guest is to mix a polar help gas with the guest species. Unfortunately, the result is a mixed or double clathrate that can obscure each other. This is particularly likely in the far-infrared where the polar molecule may have rotational lines that overlap and dominate a weaker rattling absorption of the non-polar molecule. Furthermore, the fraction of cages occupied by each species must be known to infer the absorption of the non-polar hydrate from the observed absorption.

We chose to grow a pure $\mathrm{CO}_{2}$ hydrate sample. Miller and Smythe [1970] successfully formed $\mathrm{CO}_{2}$ hydrate by pressurizing thinly divided ice, which took minutes or hours to convert. We require a slab sample a few tenths of a millimeter thick, which would no doubt require an unacceptably long formation time. We instead grew our samples from the liquid water state using a technique similar to that used by Kiefte et al. [1985].

Figure 1 shows an exploded view of the infrared cell we constructed for our experiment. It consists of copper clamps holding a pair of polyethylene windows over the ends of a short copper pipe. The windows allow far-infrared access and also act as gaskets. The lower, thicker window is also a substrate on which the clathrate forms. We milled $0.5 \mathrm{~mm}$ from the center of the window to form a shallow cup to hold liquid water.

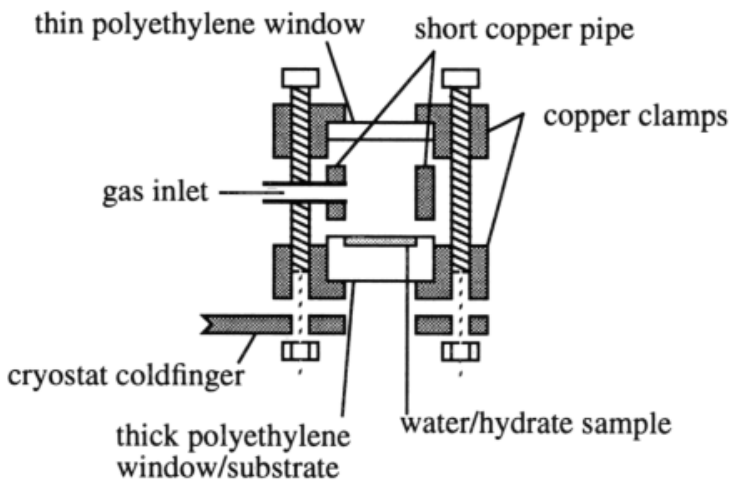

Figure 1. Exploded view of far-infrared cell

$\mathrm{CO}_{2}$ gas introduced through a copper tube pressurizes the water. Stainless steel screws clamp the cell together and to the coldfinger of a liquid nitrogen cooled cryostat. A platinum resistance temperature detector sensor in good thermal contact with the cell measures the cell temperature and provides a feedback signal to a temperature controller that varies the voltage on the cryostat heater, stabilizing the temperature to about $1 \mathrm{~K}$.

The largest uncertainty is due to uncertainty in sample thickness. We determined the thickness from the amount of water in the cell and the expansion properties of the water to hydrate phase change. At $0^{\circ} \mathrm{C}$, $\mathrm{CO}_{2}$ hydrate has a cubic unit cell containing 46 water molecules with a lattice parameter of $1.207 \mathrm{~nm}$. Using the molecular weights of the water molecules, we calculated an empty hydrate density of $0.80 \mathrm{~g} / \mathrm{cm}^{3}$. Liquid water thus expands by $25 \%$ when converting to hydrate.

Using a micropipet, we placed $40 \mu$ l of distilled, deionized water on the substrate. Based on the dimensions of the cell, this corresponds to a slab of water $0.45 \mathrm{~mm}$ thick. When converting to a hydrate, the sample should expand to $0.56 \mathrm{~mm}$. We somewhat arbitrarily place a generous $0.5 \mathrm{~mm}$ uncertainty on the thickness.

To test the evaporation rate of the water, we closed the cell, left the $\mathrm{CO}_{2}$ port valve open, and let the system sit for 16 hours. We observed no measurable loss of water. We therefore assumed that no water was lost to evaporation in the relatively short time of about one hour during which the hydrate was prepared.

We performed extensive experiments to determine a feasible method for preparing a high quality sample. Initial tests were made with a plexiglass window replacing the upper polyethylene window so that we could see the state of the sample. The cell was loaded with water, attached to the coldfinger, and pressurized with $\mathrm{CO}_{2}$ gas to 28.2 atm (415 psi), which is well above the $12.4 \mathrm{~atm}$ dissociation pressure at $0^{\circ} \mathrm{C}$ given by Davidson [1973]. The pressurized cell was slowly cooled toward $0^{\circ} \mathrm{C}$. At about $3^{\circ} \mathrm{C}$ the water froze, which is in reasonable agreement with published hydrate phase behavior [Berecz and Bal la-Achs, 1983]. The existence of the hydrate was further confirmed by reducing the pressure and observing the sample bubble profusely and melt. 
The solid, pressurized sample at $3^{\circ} \mathrm{C}$ was clear, but, as the temperature was lowered a few degrees below $0^{\circ} \mathrm{C}$, the sample became cloudy. This behavior suggests that only a thin layer at the top of the sample had converted to hydrate, and that the liquid below it was freezing into a cloudy sample of water ice at the lower temperature. To confirm this hypothesis, low resolution, far-infrared transmission measurements of the pressurized sample were made over the range $-10^{\circ} \mathrm{C}$ to $0^{\circ} \mathrm{C}$. At temperatures above about $-4^{\circ} \mathrm{C}$ the sample was quite opaque, indicating the presence of liquid water. At lower temperatures, the sample showed a spectrum with absorption increasing with frequency, which is characteristic of water ice [Mishima, et al., 1983] and clathrate far-infrared spectra [Gerbaux et al., 1975].

The hydrate layer forming at the top of the sample slowed migration of the $\mathrm{CO}_{2}$ gas to the underlying water. To effectively cool the bottom first, thin strips of copper foil were run across the substrate and thermally joined to the short copper pipe. Samples prepared in this manner did not become cloudy and were not opaque to far-infrared radiation in the $-10^{\circ} \mathrm{C}$ to $0^{\circ} \mathrm{C}$ range.

We measured transmission spectra of our samples with a Bomem DA8 Fourier transform spectrometer configured with a mercury lamp, a $100 \mu$ m mylar beamsplitter, and a liquid helium cooled silicon bolometer at $4.2 \mathrm{~K}$. Our system suffers from a $15 \mathrm{~Hz}$ mechanical vibration that introduces spurious spectral features. By changing the speed of the scanning mirror, it was possible to change the frequency at which these artificial features occurred in the spectrum, but such features could not be completely eliminated. Several speeds were tested to insure that the features did not corrupt the interesting parts of the spectrum.

Clathrate samples were cooled to $-5^{\circ} \mathrm{C}$, and the pressure was reduced to 20.4 atm (300 psi) to prevent liquid $\mathrm{CO}_{2}$ from condensing. The cell was then sealed and cooled to $150 \mathrm{~K}$, the temperature of the Martian pole in winter, and another spectrum was measured. The spectrum was ratioed with the spectrum of the empty cell at the same temperature to form a transmission spectrum. Significant amounts of $\mathrm{CO}_{2}$ ice did not accumulate in the spectrometer beam path because there was no evidence of the $68 \mathrm{~cm}^{-1} \mathrm{CO}_{2}$ ice lattice absorption.

\section{Discussion}

The solid curve in Figure 2 is the transmission spectrum of the hydrate sample. The decrease at higher frequencies is characteristic of other hydrate and water ice measurements. There is a clear low frequency absorption band that spans from about $22 \mathrm{~cm}^{-1}$ to below the minimum frequency our system could reliably measure. The position and the weak two lobe structure are reproducible between measurements. The absorption is probably due to a rattling mode of the $\mathrm{CO}_{2}$ molecule because of its low frequency and the lack of pure $\mathrm{CO}_{2}$ rotational transitions.

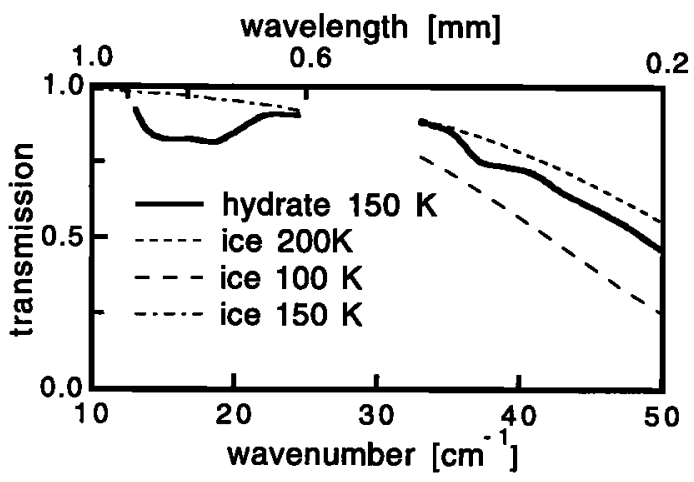

Figure 2. Transmission spectra of hydrate with ice data for comparison

The apparent feature at $37 \mathrm{~cm}^{-1}$ moves with mirror speed and is an artifact caused by mechanical vibration. The data between $25 \mathrm{~cm}^{-1}$ and $33 \mathrm{~cm}^{-1}$ is unreliable because the $100 \mu \mathrm{m}$ mylar beamsplitter is ineffective in this region. Crude measurements made with a thinner beamsplitter showed no features in this gap.

Figure 2 also shows transmission data of $0.56 \mathrm{~mm}$ water ice samples for comparison. The higher frequency curves are based on measurements made of $100 \mathrm{~K}$ and $200 \mathrm{~K}$ ice samples by Whalley and Labbe [1969]. The transmission spectrum of an ice sample of $150 \mathrm{~K}$ ice presumably would lie between the $100 \mathrm{~K}$ and $200 \mathrm{~K}$ curves and would be similar to the clathrate sample. The lower frequency ice curve is based on measurements and theory by Mishima et al. [1983] of $150 \mathrm{~K}$ water ice. The nearness of our transmission curve to the ice curves show that the assumed thickness of the sample is plausible. The ice transmission clearly does not exhibit absorption features in this region of the far-infrared. $\mathrm{CO}_{2}$ gas and $\mathrm{CO}_{2}$ ice do not have strong absorption here either. (We know of no far-infrared data for $\mathrm{CO}_{2}$ ice away from its lattice absorptions. This is probably because the attenuation is very weak and thick samples are difficult to make. See Warren's review [1985].) The hydrate absorption is unique and may give rise to a distinctive radiometric signature.

To model the emission from a Martian hydrate layer, we must know the complex hydrate index of refraction or, equivalently, the dielectric constant. The dielectric constants of structure I and II hydrates have been measured in the water molecule and guest molecule relaxation regions [Davidson, 1973, p. 183]. For nonpolar guests, the dielectric relaxation spectra asymptotically approach 2.9 with increasing frequency. Since there should be no strong absorptions in these hydrates at higher frequencies until the lattice starts absorbing, the dielectric value of 2.9 should extend into the farinfrared. We therefore assume the real part of the dielectric is 2.9, which corresponds to a real index of 1.7 .

We used the measured transmission spectrum to estimate the imaginary index. For this, we considered reflections at the hydrate-substrate and hydrate-gas interfaces. We determined the index of refraction of the 


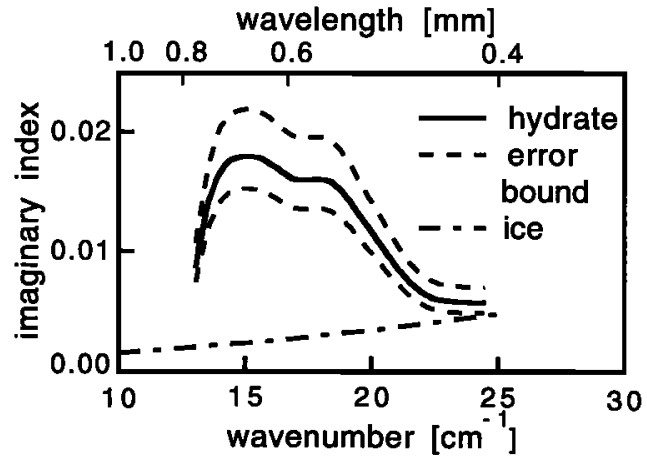

Figure 3. Imaginary indices of refraction of hydrate with ice data for comparison

substrate to be 1.6. Since this is so close to the index of the hydrate, we neglect reflections at the clathratesubstrate interface. Furthermore, the reflection at the clathrate-gas interface in the sample measurement is similar to the reflection at the substrate-gas interface in the reference measurement. We thus assume that these losses cancel out when the raw sample and reference spectra are ratioed to form the transmission spectrum.

These simplifications lead to a transmission

$$
T=e^{-4 \pi \sigma_{0} n_{1} d}
$$

where $\sigma_{0}$ is the free space wavenumber, $n_{i}$ is the imaginary index, and $d$ is the sample thickness. The imaginary index is obtained by

$$
n_{i}=-\frac{\ln (T)}{4 \pi \sigma_{0} d}
$$

Figure 3 gives the spectrum of the imaginary index for the hydrate at $150 \mathrm{~K}$ as well as that of ice, as reported by Mishima et al. [1983]. The dotted lines above and below the hydrate curve are error bounds resulting form the $\mathrm{mm}$ uncertainty in the hydrate sample thickness.

Whether or not the observed absorption is sufficiently strong to provide an unambiguous radiometric signature depends upon the physical characteristics of the hydrate layer being measured. Kieffer [1991] has modeled the Martian residual cap and predicts particle sizes of $100 \mu \mathrm{m}$ or more. Our preliminary modeling of emission from a layer of Mie scattering dielectric spheres shows that for average particle diameters greater than $100 \mu \mathrm{m}$, the brightness temperature at $17 \mathrm{~cm}^{-1}$ of a layer of hydrate particles is $10 \mathrm{~K}$ or more higher than at $24 \mathrm{~cm}^{-1}$. The temperature difference for ice particles is about a factor of ten lower. The complete results of the modeling will be presented in a future paper, where the possibility of ground-based or spaceborne measurements will be also be discussed.

\section{References}

Berecz, E. and M. Balla-Achs, Gas Hydrates, Elsevier, New York, 1983.
Bertie, J. E., and E. Whalley, Infrared Spectra of Ices Ih and Ic in the Range 4000 to $350 \mathrm{~cm}^{-1}, J$. Chem. Phys., 40, 1637-1645, 1964.

Bertie, J. E., and E. Whalley, Optical Spectra of Orientationally Disordered Crystals. II. Infrared Spectrum of Ice Ih and Ice Ic from 360 to $50 \mathrm{~cm}^{-1}$, J. Chem. Phys., 46, 1271-1284, 1967.

Davidson, D. W., Clathrate Hydrates, in Water: $A$ Comprehensive Treatise, F. Franks, Ed., vol. 2, pp. 115-234, Plenum Press, New York, 1973.

Fleyfel, F. and J. P. Devlin, FT-IR Spectra of $90 \mathrm{~K}$ Films of Simple, Mixed, and Double Clathrate Hydrates of Trimethylene Oxide, Methyl Chloride, Carbon Dioxide, Tetrahydrofuran, and Ethylene Oxide Containing Decoupled $\mathrm{D}_{2} \mathrm{O}, J$. Phys. Chem., 92, 631-635, 1988.

Gerbaux, M. M., C. Barthel, and A. Hadni, Absorption de Clathrates de Glace dans L'infrarouge Lointain, Spectrochimica Acta, 31A, 1901-1903, 1975.

Handa, Y. P. and J. G. Cook, Thermal Conductivity of Xenon Hydrate, J. Phys. Chem., 91, 6327-6328, 1987.

James, P. B., H. H. Kieffer, and D. A. Paige, The Seasonal Cycle of Carbon Dioxide on Mars, in Mars, Kieffer et al., Eds., pp. 934-968, Univ. of Arizona Press, Tucson, 1992.

Kieffer, H. H., $\mathrm{H}_{2} \mathrm{O}$ Grain Size and the Amount of Dust in Mars' Residual North Polar Cap, J. Geophys. Res., 95, 1481-1493, 1990.

Kiefte, H., M. J. Clouter, and R. E. Gagnon, Determination of Acoustic Velocities of Clathrate Hydrates by Brillouin Spectroscopy, J. Phys. Chem., 89, 3103-3108, 1985.

Miller, S. L., Clathrate Hydrates in the Solar System, in Ices in the Solar System, J. Klinger et al., Eds., pp. 59-78, D. Reidel, Boston, 1985.

Miller, S. L. and W. D. Smythe, Carbon Dioxide Clathrate in the Martian Polar Cap, Science, 170, pp. 531-533, 1970.

Mishima, O., D. D. Klug, and E. Whalley, "The Far-infrared Spectrum of Ice Ih in the Range 8-25 $\mathrm{cm}^{-1}$ : Sound Waves and Difference Bands, with Application to Saturn's Rings, J. of Chem. Phys., 78, 6399-6404, 1983.

Pouchert, C. J. (Editor), The Aldrich Library of FT-IR Spectra, edition 1, volume 3: Vapor Phase, Aldrich Chemical Co., Milwaukee, 1989.

Richardson, H. H., P. J. Woolridge, and J. P. Devlin, FTIR Spectra of Vacuum De posited Clathrate Hydrates of Oxirane, $\mathrm{H}_{2} \mathrm{~S}$, THF, and Ethane, J. Chem. Phys., 83, 4387-4394, 1985.

Smythe, W. D., Spectra of Hydrate Frosts: Their Application to the Outer Solar System, Icarus, 24, 421-427, 1975.

Tse, J. S. and M. L. Klein, Computer simulation studies of the structure I clathrate hydrates of methane, tetraflouromethane, cyclopropane, and ethylene oxide, $J$. Chem. Phys., 81, 6146-6153, 1984.

Warren, S. G., Optical Constants of Carbon Dioxide Ice, Applied Optics, 25, 2650-2674, 1986.

Whalley, E. and H. J. Labbe, Optical Spectra of Orientationally Disordered Crystals. III. Infrared Spectra of the Sound Waves, J. Chem. Phys., 51, 3120-3127, 1969.

J. C. Landry and A. W. England, Radiation Laboratory, 1301 Beal Avenue, Ann Arbor, MI 48109-2122.

jclandry@eecs.umich.edu and england@eecs.umich.edu

(received July 29, 1994; accepted September 19, 1994.) 